\title{
A Robust Detection Method of Short Atrial Fibrillation Episodes
}

\author{
Zouhair Haddi ${ }^{1}$, Jean-François Pons ${ }^{2}$, Stéphane Delliaux ${ }^{3,4}$, Bouchra Ananou ${ }^{1}$, Jean-Claude \\ Deharo $^{3,5}$, Ahmed Charaï ${ }^{2}$, Rachid Bouchakour ${ }^{2}$, Mustapha Ouladsine ${ }^{1}$ \\ ${ }^{1}$ Aix Marseille Univ., Univ. Toulon, CNRS, ENSAM, LSIS, Marseille, France \\ ${ }^{2}$ Aix Marseille Univ., Univ. Toulon, CNRS, IM2NP, Marseille, France \\ ${ }^{3}$ Aix Marseille Univ., IRBA, DS-ACI, Marseille, France \\ ${ }^{4}$ APHM, Hôpital Nord, Service des Explorations Fonctionnelles Respiratoires, Pôle cardiovasculaire, \\ Marseille, France \\ ${ }^{5}$ APHM, Hôpital La Timone, Service de Cardiologie du pôle cardiovasculaire et thoracique, \\ Marseille, France
}

\begin{abstract}
This study aimed to detect short-duration Atrial Fibrillation (AF) episodes by studying inter-beat interval time series to reach real-time automatic medical monitoring. Four publicly-accessible sets of clinical data were used for assessment. All time series were segmented in 1-min RR interval windows and then three specific features were calculated, namely, Vector Angular Index, Vector Length Index and Dispersion of points along the perpendicular to the diagonal line. The features of the four databases were merged in order to give rise to huge variability and therefore to better characterize $A F$ rhythm. Principal Component Analysis (PCA) was used to elucidate whether it was possible to discriminate between AF and Normal Sinus Rhythm (NSR) and Learning Vector Quantization (LVQ) neural network has been optimized to develop the classification model. PCA analysis has shown a good discrimination between the studied rhythms. Furthermore, despite its very simple structure, $L V Q$ neural network has performed better on the analysed databases than existing algorithms did, with high sensitivity and specificity respectively of $99.19 \%$ and 99.39\%.
\end{abstract}

\section{Introduction}

Atrial fibrillation (AF), considered as the most common cardiac arrhythmia, is a major public health burden associated with significant morbidity and mortality [1]. In recent years, several algorithms have been developed to detect AF specifically when short duration occurs [2]. Most of them are based on inter-beat interval time series analysis. Although progress in the published results is seen, there is still scope for improvement which needs to be addressed especially for brief duration as short as one minute or less. In this context, Langley et al. [3], have evaluated three features, namely, coefficient of variation, mean successive difference and coefficient of sample entropy for a recording duration of only $10 \mathrm{~s}$. Sensitivities of greater than $94 \%$ and specificities of around $93 \%$ were achieved. Other assay of entropy was conducted by D.E. Lake et al. [4], in order to assess short AF episodes of 12 beats. Optimal template length and tolerance matching have been carefully checked. The receiver operating characteristic analysis results allowed to reach a sensitivity of $91 \%$ and a specificity of $94 \%$ for the MIT$\mathrm{BIH}$ dataset. S. Hargittai has investigated the performance of several features, extracted from segments of 80 beats to detect AF [5]. He confirmed that the use of the scatter plot of successive RR differences (dRR Lorenz Plot) and Sample Entropy yielded an overall error rate of about 5\% for Physionet datasets (MIT-BIH arrhythmia, atrial fibrillation and long-term atrial fibrillation databases). In the current study, three geometrical features were used, namely, Vector Angular Index (VAI), Vector Length Index (VLI), and dispersion of points along the perpendicular to the diagonal line (SD1) have been exploited as input to PCA and LVQ to diagnosis AF of 1min episode. These parameters have been previously employed to detect $\mathrm{AF}$ [6] by using univariate analysis and were tested on only 60 recordings of AF Termination Challenge Database (80 recordings).

\section{Methods}

\subsection{Databases}

PCA and LVQ were evaluated on four publiclyaccessible sets of clinical data: AF Termination Challenge 
Database, MIT-BIH AF, Normal Sinus Rhythm RR Interval Database, and MIT-BIH Normal Sinus Rhythm Databases. All time series were segmented in 1-min RR interval windows (total of 47156 and 4902 time series for NSR and AF respectively). Usually, authors trained their algorithms on one of these datasets and tested them on the remaining. We believe that if the four datasets are merged (and categorized into AF and NSR groups), this could give rise to huge variability and therefore to better characterize AF rhythm. To illustrate this idea, we have calculated Vector Angular Index values for AF Termination Challenge Database (Figure 1 (a)) and compared them with those of 1-min AF episodes of the merged datasets (Figure 1 (b)). As it can be seen, there is a clear variability has emerged from the merged datasets.

\section{2. $\quad$ R-R time series features}

Three features were extracted from the scatter plot (defined as a diagram in which each R-R interval is
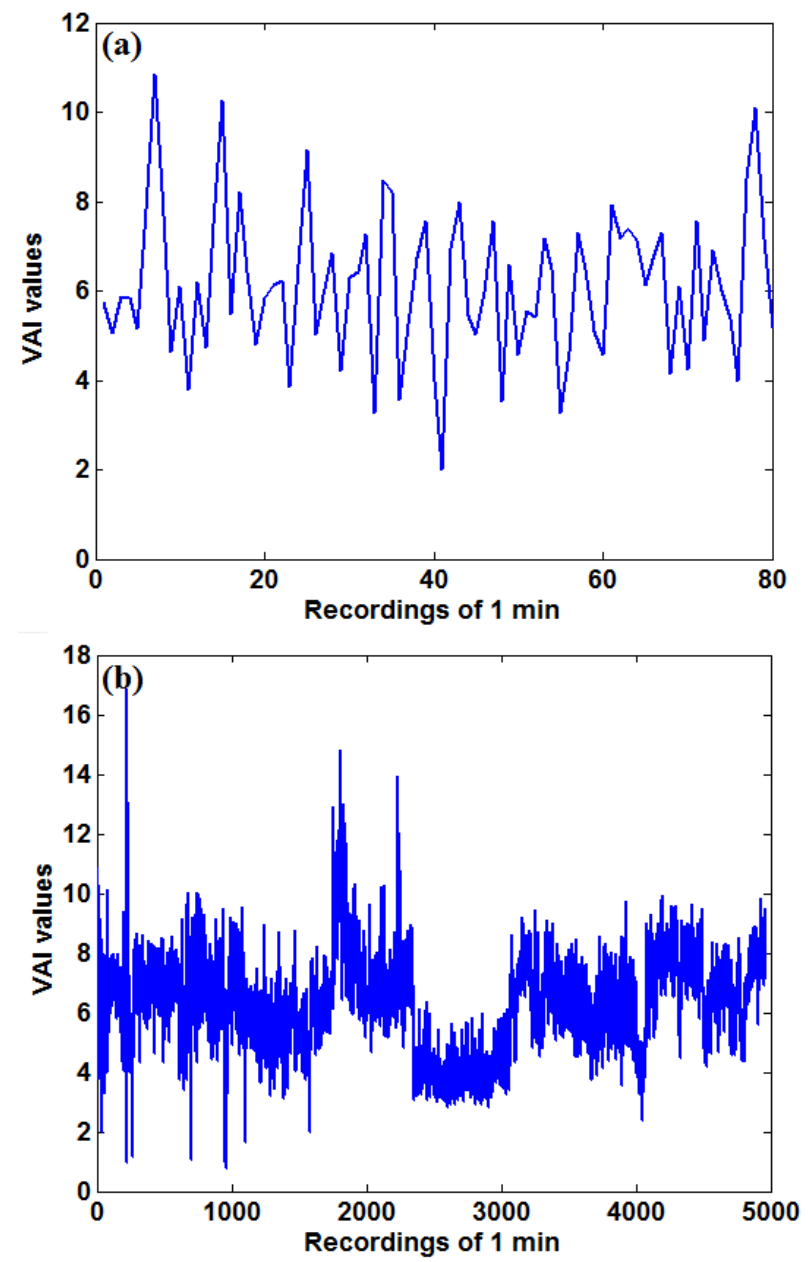

Figure 1. VAI calculated for AF Termination Challenge Database (a) and for 1-min AF episodes of the merged databases (b).
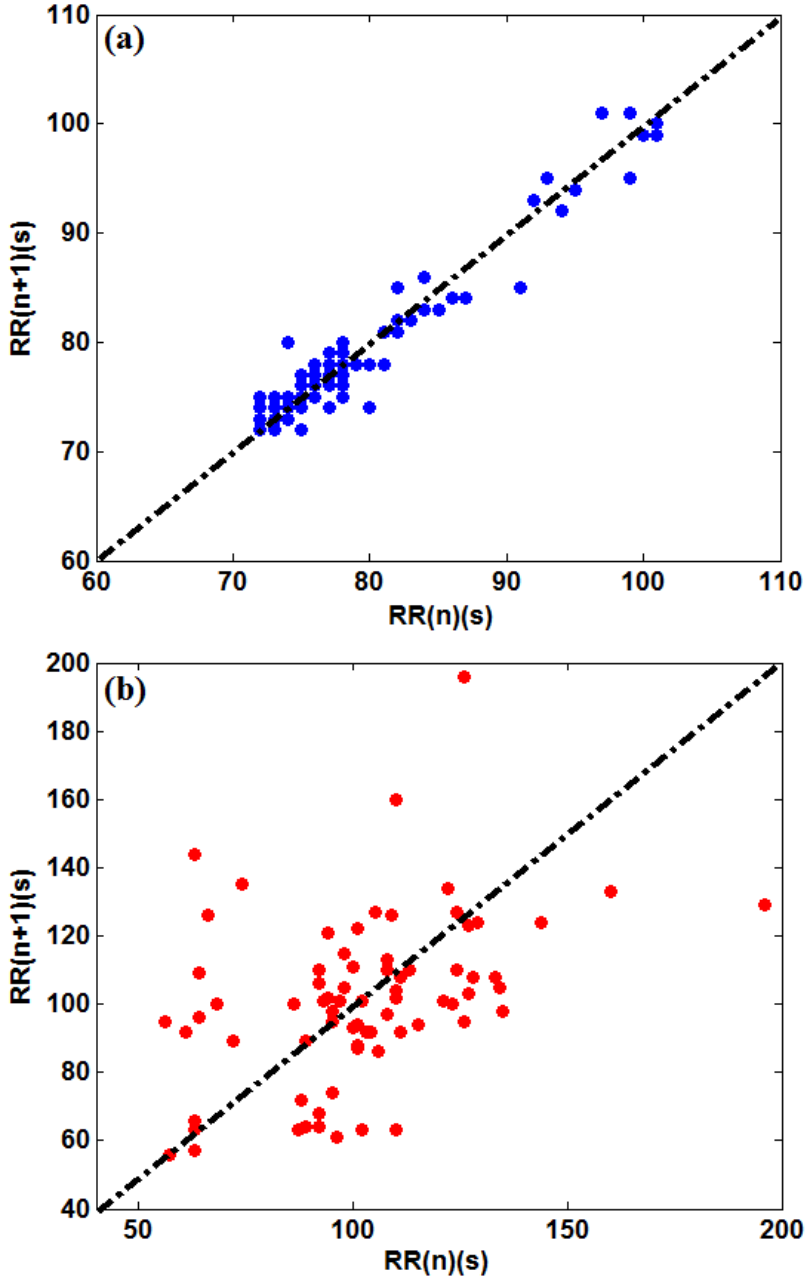

Figure 2. Scatter plots of R-R interval signal of NSR (a), and $\mathrm{AF}(\mathrm{b})$.

plotted as a function of the previous R-R interval) to classify AF from NSR. The scatter plot of NSR seems as sticky, in that nearly all the scatter points are centralized along the diagonal line, as shown in Figure 2 (a). However, the scatter plot of AF seems as unfolded fanshaped, in which all the points are dispersed around the whole plot, as shown in Figure 2 (b). These features, which were first used for univariate analysis by X. Ruan et al. [6] are described as follows:

VAI is calculated as the mean of all the absolute value of angular differences between the lines plotted from every scatter point to the original point and the diagonal line, measuring the angular dispersion of all the points. VLI is calculated as the standard deviation of all distances of scatter points from the original point, measuring the distance dispersion of all the points. They can be defined as:

$$
V A I=\frac{\sum_{i=1}^{N}\left|\theta_{i}-45\right|}{N}
$$




$$
V L I=\frac{\sqrt{\sum_{i=1}^{N}\left(l_{i}-L\right)^{2}}}{N}
$$

where $\theta_{i}$ is the angle between the line plotted from every scatter point to the original point and the x-axis, $l_{i}$ is length between every scatter point and the original point, $L$ is the mean of all the $\mathrm{l}_{\mathrm{i}}, N$ is the number of scatter points.

SD1 is calculated as the standard deviation of the distances of points from $y=x$ axis, measuring the width of the ellipse and indicating the short-term variability and is defined as:

$$
S D 1=S T D\left(\frac{\left|R R_{n+1}-R R_{n}\right|}{\sqrt{2}}\right)
$$

where $R R_{n}$ is an R-R interval series with $n=1,2, \ldots, N-1$, $R R_{n+1}$ is the same as $\mathrm{RR}_{n}$ index-shifted by 1 and $\operatorname{STD}(x)$ represents the standard deviation of $x$.

\subsection{Multivariate data analysis}

PCA is a very well-known unsupervised method often employed in ECG signal processing [7]. The main objective of PCA consists in expressing the information contained in a dataset by a smaller number of variables called principal components. These principal components are linear combinations of the original response vectors. The principal components are chosen to contain the maximum data variance and to be orthogonal. Hence, PCA allows the reduction of multidimensional data to a lower dimensional approximation, while simplifying the interpretation of the data by the first two or three principal components ( $\mathrm{PC} 1, \mathrm{PC} 2$, and $\mathrm{PC} 3$ ) in two or three dimensions and preserving most of the variance in the data [8].

LVQ, introduced by Kohonen [9], is one of prominent learning based algorithms of artificial neural network. This algorithm and its variants have been intensively studied because of their robustness, adaptivity and efficiency. The idea of LVQ is to define class boundaries based on prototypes, a nearest neighbor rule and a winner-takes-all paradigm. The standard LVQ tries to adjust the weights using heuristic error correction rules by minimizing an objective function. The LVQ results are strongly dependent on the initial positions of the prototypes [10].

\section{Results and discussion}

\subsection{PCA analysis results}

The PCA score plot was used as an exploratory technique to investigate clustering of data points within the multi-dimensional space of features. The variables were organized in a rectangular matrix as a database. A mean-centering pre-processing technique was applied to the dataset [11]. Figure 2 (a) shows the projections of the 1-min episodes of AF and NSR on a two-dimensional scheme formed by the first two principal components. The values of $89.02 \%$ data variance explained by the first PC and $09.41 \%$ of data variance explained by the second PC indicate the importance of the first one for pattern separation. This means that the differences existing among AF and NSR episodes along the first axis are more significant than those existing along the second axis. Even if Figure 3 (a) does not show very clear separable groups, Figure 3 (b), which is a zoom-in of the groups borders, demonstrated that a powerful pattern recognition tool could discriminate between AF and NSR rhythm.

\subsection{LVQ neural network results}

PCA was performed to elucidate whether it was possible to discriminate AF and NSR groups, however, this method cannot be used as proper identification tools.
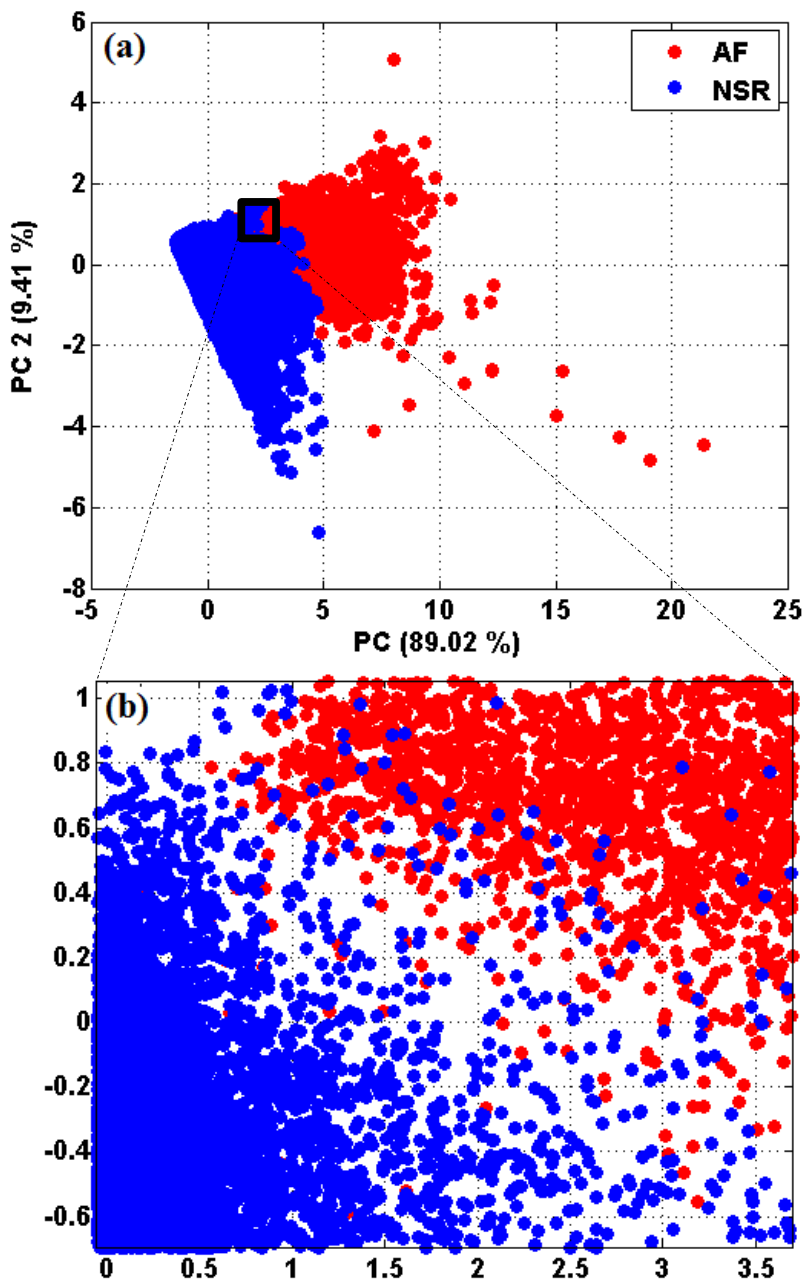

Figure 3. Scores plot of PCA performed on AF and NSR of 1-min episodes. 
Since the aim of this work was to identify AF episodes of short duration, LVQ neural network was applied to develop the classifier model. The initial weights of LVQ network were generated randomly just before the learning phase began. Very simple structure (3 neurons for AF and 1 for NSR) has been found to yield very good findings. Since results may be influenced by the selection of the test and training sets, ten test sets $(n=17373)$ were randomly selected. Finally, the selected LVQ classifier has reached a $99.37 \%$ success rate in the identification of the AF and NSR groups. Table 1 shows the confusion matrix of the LVQ classifier. Rows indicate true categories and columns predicted categories. As it can be noticed in this table, of 52118 1-min segments of AF and NSR, only 327 mistakes occurred: 40 1-min episodes belonging to $\mathrm{AF}$ were misclassified as belonging to NSR, and 287 1-min episodes belonging to NSR were misclassified as belonging to AF. In other words, $99.19 \%$ and $99.39 \%$ have been reached as sensitivity and specificity respectively.

Table 1. LVQ classification results.

\begin{tabular}{lll}
\hline \multirow{2}{*}{ Actual } & Predicted & \\
\cline { 2 - 3 } & AF & NSR \\
\hline AF & 4862 & 40 \\
NSR & 287 & 46869 \\
\hline
\end{tabular}

\section{Conclusion}

In this paper, both the PCA and the LVQ neural network classifier are presented as diagnostic tools to facilitate medical decision making in the analysis of $\mathrm{AF}$ arrhythmia. The method is based on the analysis of RRinterval time series extracted from ECG recordings. The automated AF detection holds several interesting properties, and can be implemented with only few arithmetical operations which makes it a suitable choice for telecare applications. For future works, we attempt to detect very short AF episodes (less than one min) from $\mathrm{RR}$ interval signals.

\section{Acknowledgements}

This work is part of the APPRISE/HIT project and was performed with the support of the $A^{*}$ MIDEX project $\left(n^{\circ}\right.$ ANR-11-IDEX-0001-02) funded by the "Investissements d'Avenir" program of the French Government, which is managed by the French National Research Agency (ANR).

\section{References}

[1] Ponikowski P, Voors AA, Anker SD, Bueno H, Cleland JGF, Coats AJS, Falk V, Gonzalez-Juanatey JR, Harjola V-
P, Jankowska EA, Jessup M, Linde C, Nihoyannopoulos P, Parissis JT, Pieske B, Riley JP, Rosano GMC, Ruilope LM, Ruschitzka F, Rutten FH, Meer PVD. ESC Guidelines for the diagnosis and treatment of acute and chronic heart failure: The Task Force for the diagnosis and treatment of acute and chronic heart failure of the European Society of Cardiology (ESC). Developed with the special contribution. Eur. J. Heart Fail. 2016;18:891-975.

[2] Pons J-F, Haddi Z, Deharo J-C, Charaï A, Bouchakour R, Ouladsine M, Delliaux S. Heart rhythm characterization through induced physiological variables. Scientific Reports 2017;5059:1-13.

[3] Langley P, Dewhurst M, Di Marco LY, Adams P, Dewhurst F, Mwita JC, Walker R, Murray A. Accuracy of algorithms for detection of atrial fibrillation from short duration beat interval recordings. Medical Engineering \& Physics 2012;34:1441-1447.

[4] Lake DE, Moorman JR. Accurate estimation of entropy in very short physiological time series: the problem of atrial fibrillation detection in implanted ventricular devices. Am J Physiol Heart Circ Physiol 2011;300:H319-H325,

[5] Hargittai S. Is it Possible to Detect Atrial Fibrillation by Simply using RR Intervals?. Computing in Cardiology 2014;41:897-900.

[6] Ruan X, Liu C, Wang X, Li P. Automatic Detection of Atrial Fibrillation Using R-R Interval Signal, Conf. Proc of 4th International Conference on Biomedical Engineering and Informatics (BMEI) 2011; 644-647.

[7] Castells F, Laguna P, Sörnmo L, Bollmann A, Roig JM. Principal Component Analysis in ECG Signal Processing. EURASIP Journal on Advances in Signal Processing 2007;74580:1-21.

[8] Bougrini M, Tahri K, Haddi Z, Saidi T, El Bari N, Bouchikhi B. Detection of Adulteration in Argan Oil by Using an Electronic Nose and a Voltammetric Electronic Tongue. Journal of Sensors 2014;245831:1-10.

[9] Kohonen, T. Learning vector quantization for pattern recognition. Technical Report 1986;TKK-F-A601.

[10] Boubezoul A, Paris S, Ouladsine M. Application of the cross entropy method to the GLVQ algorithm. Pattern Recognition 2008;41:3173-3178.

[11] Haddi Z, El Barbri N, Tahri K, Bougrini M, El Bari N, Llobet E, Bouchikhi B. Instrumental assessment of red meat origins and their storage time using electronic sensing systems. Analytical Methods 2015;7:5193-5203.

Address for correspondence.

Zouhair Haddi

Laboratoire des Sciences de l'Information et des Systèmes (LSIS)

Domaine Universitaire de Saint-Jérôme

Avenue Escadrille Normandie-Niemen

13397 Marseille, France.

zouhair.haddi@1sis.org 